Peer Reviewed Paper openaccess

\title{
Unstained blood smear contrast enhancement using spectral time multiplexing super resolution
}

\author{
Marie-Florence A. Yebouet, ${ }^{\mathrm{a}, *}$ Ambroise K. Diby, ${ }^{\mathrm{b}}$ Kenneth A. Kaduki ${ }^{\mathrm{c}}$ and Jérémie T. Zoueu ${ }^{\mathrm{d}}$ \\ aLaboratoire d'Instrumentation, Image and Spectroscopie, Institut National Polytechnique Félix Houphouët-Boigny, Yamoussoukro, Côte \\ d'Ivoire and Laboratoire de Physique de la Matière Condensée et Technologie, Université Félix Houphouët-Boigny, Abidjan, Côte d'Ivoire. \\ E-mail: mafloh2@yahoo.fr \\ 'Laboratoire de Physique de la Matière Condensée et Technologie, Université Félix Houphouët-Boigny, Abidjan, Côte d'Ivoire \\ 'Department of Physics, University of Nairobi, PO Box 30197 - 00100 , Nairobi, Kenya \\ 'Laboratoire d'Instrumentation, Image and Spectroscopie, Institut National Polytechnique Félix Houphouët-Boigny, Yamoussoukro, Côte d'Ivoire \\ ORCID iDs \\ M.-F. Yebouet: https://orcid.org/0000-0001-9954-7484 \\ A. Diby: https://orcid.org/0000-0001-7046-1150 \\ K. Kaduki: https://orcid.org/0000-0002-0394-6999 \\ J. Zoueu: https://orcid.org/0000-0003-4588-1641
}

\begin{abstract}
We report the use of Time Multiplexing Super Resolution (TMSR) to reduce significantly speckle noise in spectral imaging microscopy of unstained thin blood smear samples of malaria-infected blood. The method is based on combining speckle illumination with a moving array serving as an encoding mask. We propose the use of a new encoding mask to improve the performance of the conventional TMSR method. The new mask is a two-dimensional generalisation of the one-dimensional Ipatov code. The mask is projected on the object and 13 low-resolution images captured and subsequently decoded properly using the same array. The low contrast images are added and extracted from the resulting reconstruction, giving a super-resolved, high-contrast image. The Ipatov filter used in this work performs better than the Barker filter.
\end{abstract}

Keywords: image contrast, time multiplexing, transfer function, super resolution, image processing, blood smear, multispectral, speckle reduction, diffraction, malaria

\section{Introduction}

Malaria is the leading cause of death in Africa. However, the malaria diagnosis method remains manual and time consuming. In the microscopy of blood smear samples, combining spectral imaging and image processing can lead to faster and more accurate diagnoses of diseases such as malaria. However, the coherent nature of lasers used in sample illumination leads to low contrast images, due to the addition of speckle patterns in the red blood cell images. Spatial resolution in optical microscopy is also limited by diffraction. ${ }^{1}$ Several techniques have been developed to reduce speckle noise and improve spatial resolution. Time multiplexing super resolution (TMSR),

\section{Correspondence}

Marie-Florence Yebouet (mafloh2@yahoo.fr)

Received: 30 September 2019

Revised: 25 November 2019

Accepted: 25 November 2019

Publication: 1 January 2020

doi: 10.1255/jsi.2020.a1

ISSN: 2040-4565

\author{
Citation \\ M.-F.A. Yebouet, A.K. Diby, K.A. Kaduki and J.T. Zoueu, "Unstained \\ blood smear contrast enhancement using spectral time multiplexing super \\ resolution", J. Spectral Imaging 9, a1 (2020). \\ https://doi.org/10.1255/jsi.2020.a1 \\ (C) 2020 The Authors \\ This licence permits you to use, share, copy and redistribute the paper in \\ any medium or any format provided that a full citation to the original \\ paper in this journal is given, the use is not for commercial purposes and \\ the paper is not changed in any way.


for example, produces images that are resolved beyond the diffraction limit.

The original concept of TMSR suggested by Françon ${ }^{2}$ uses two scanning pinholes to encode and decode the information embedded in the object. One pinhole is placed on the plane of the object to encode the information, while the other decodes the information on the image plane. Other approaches have been developed using different masks to improve the spatial resolution of images. ${ }^{3-9}$ The nature and properties of the mask play an important role in image reconstruction. Some approaches have taken advantage of speckle illumination to improve spatial resolution in optical microscopy. In this paper, we propose a binary transmission 2D Ipatov-based array as an encoding and decoding mask for image reconstruction. The method relies on the autocorrelation (AC) of the mask.

Multispectral microscopy is a technique that exploits spectral and spatial information resulting from lightmatter interaction. Several studies have reported the use of multispectral microscopy in the detection of malaria without fluorescent labelling of cells ${ }^{10,11}$ and the early detection of plant diseases. ${ }^{12,13}$ However, there is a need to improve spatial resolution and noise performance in these systems. We, therefore, present an implementation of a TMSR-based microscopy system that seeks to improve thin blood smear image contrast via a multispectral approach.

\section{Materials and methods \\ Experimental setup}

The experimental setup (Figure 1) is a laboratory-assembled optical horizontal microscope configured to work in transmission mode. The sample object was a thin blood smear illuminated by two wavelengths: $\lambda=405 \mathrm{~nm}$ and $\lambda=638 \mathrm{~nm}$. The light was structured with two different gratings: a Barker grating and the proposed Ipatov grating.

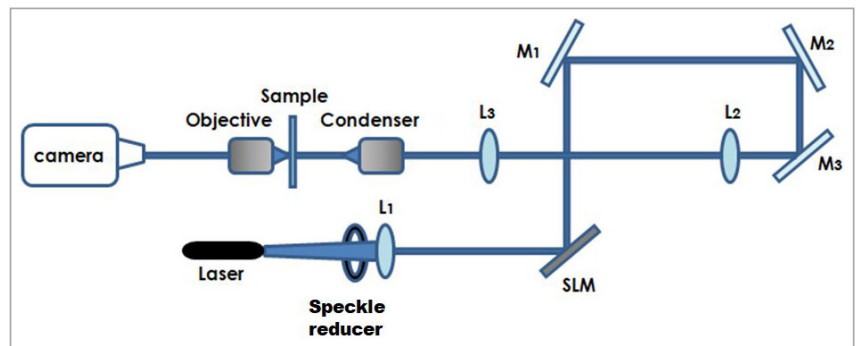

Figure 1. Experimental setup: L, lens; M, mirror; SLM, spatial light modulator.
The mask was projected on the sample and shifted laterally using the spatial light modulator (SLM) (Holoeye LC-R720). An 8-bit monochrome 1024×1280-pixel CMOS camera (Thorlabs DCC1545M) with a pixel size of $5.2 \times 5.2 \mu \mathrm{m}$ was used to acquire the images. Thirteen low-resolution images were recorded for different positions of the mask for each wavelength.

\section{Sample preparation}

For the study of red blood cells, thin blood smears were prepared on silica slides measuring $75 \times 25 \times 1 \mathrm{~mm}$ using the standard procedure. ${ }^{14}$ In conventional blood smear preparation, cells are labelled with May-Grünwald Giemsa (MGG). However, our samples were made without MGG. Unstained blood smears are preferred for microscopic imaging because they preserve the intrinsic optical properties of red blood cell components for analysis.

\section{Ipatov-based array}

A binary transmission 1D Ipatov-based code is used in radar systems. ${ }^{15}$ The longest reported Ipatov code is 13 bits long [1110010111110]. This code exhibits a twovalued cyclic AC and can be generalised into a 2D array as follows. Each row in the array is shifted five pixels to the right relative to the previous row. ${ }^{9}$ This gives the pattern shown in Figure 2(a). The resulting cyclic AC of the $13 \times 13$ array is shown in Figure $2(b)$. The cyclic AC has 13 peaks equal to 1 , with a distance of $(13)^{1 / 2}$ separating every two peaks.

\section{Measurement procedure}

The main idea was to capture blood smear images for different phases of the mask. The phase shifts were

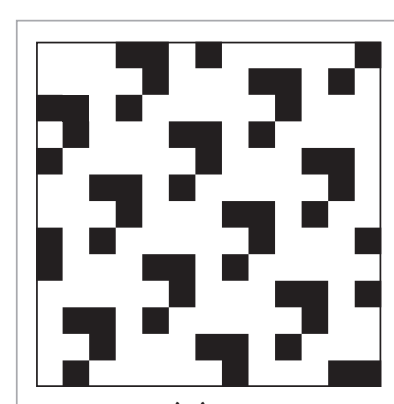

(a)

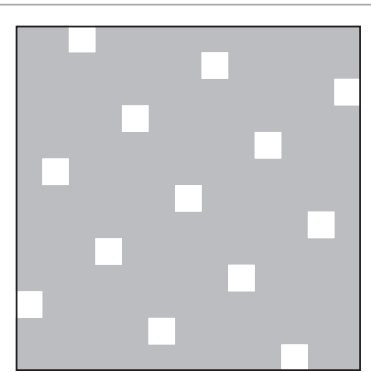

(b)
Figure 2. (a) $13 \times 13$ Ipatov-based array, where each row is a five-pixel shift of the previous row; (b) cyclic AC of the $13 \times 13$, Ipatov-based array. Pixel values: white, 1 ; grey, 2/3; black, 0 . 
achieved by moving the mask in the lateral direction using a SLM. This was done under electronic control to ensure speedy and precise image acquisition. The sample was kept static at all times.

The images were acquired and processed as follows. A blood smear image was recorded for the first position of the mask. This position was taken to be the reference position for all translations of the mask. The mask was then shifted by $20^{\circ}$ using the SLM and another image recorded. These steps were repeated for mask phase values between $0^{\circ}$ and $240^{\circ}$ in increments of $20^{\circ}$, giving a total of 13 low-resolution images. Each of the images was multiplied by the previous mask, and the resulting images were added together for reconstruction. An image of the sample without the mask was also recorded. This entire process was replicated for each of the wavelengths (405 $\mathrm{nm}$ and $638 \mathrm{~nm}$ ).

\section{Time multiplexing super resolution technique}

This technique uses two gratings. One was placed in front of the object $\left[s_{1}(x, y)\right]$ to encode the information and the other near the detector or added digitally through an algorithm $\left[s_{2}(x, y)\right]$ for decoding. During the process, 13 low-resolution images were acquired as the mask was shifted in a lateral direction. These images were then added and removed from the reconstruction to give a high-resolution image. The technique was applied for each of the two wavelengths. i.e. $405 \mathrm{~nm}$ and $638 \mathrm{~nm}$.

If $\varepsilon$ represents the displacement of the mask, we define $\mathrm{O}_{\varepsilon}(x, y)$ to be the intensity for one single frame in the camera, $g(x, y)$ the input object intensity, $O(x, y)$ the reconstructed image and $h(x, y)$ the point spread function (PSF). We then have: ${ }^{9}$

$$
\begin{aligned}
& \mathrm{O}_{\varepsilon}(x, y, t)=\left[\mathrm{g}(x, y) \mathrm{s}_{1}(x-\varepsilon t, y)\right] * h(x, y) \\
& \mathrm{O}_{\varepsilon}(x, y, t)= \\
& \iint_{-\infty}^{\infty} g\left(x^{\prime}, y^{\prime}\right) s_{1}\left(x^{\prime}-\varepsilon t, y^{\prime}\right) h\left(x-x^{\prime}, y-y^{\prime}\right) d x^{\prime} d y^{\prime}
\end{aligned}
$$

where * denotes convolution.

The decoding process involves multiplying each image by the decoding mask $s_{2}$, followed by progressive integration over the time.

$$
O(x, y)=\int_{-\infty}^{\infty} O_{\varepsilon}(x, y, t) s_{2}(x-\varepsilon t, y) d t
$$

$$
\begin{aligned}
& O(x, y)=\iiint_{-\infty}^{\infty} g\left(x^{\prime}, y^{\prime}\right) s_{1}\left(x^{\prime}-\varepsilon t, y^{\prime}\right) \ldots \ldots \\
& \ldots \ldots h\left(x^{\prime}-x^{\prime}, y-y^{\prime}\right) s_{2}(x-\varepsilon t, y) d t \cdot d x^{\prime} d y^{\prime}
\end{aligned}
$$

Since the masks are time-dependent, and assuming $\mathrm{s}_{1}$ and $s_{2}$ are the same array (i.e., $s_{1}=s_{2}=M$ ), we have:

$$
\begin{gathered}
\int_{-\infty}^{\infty} s_{1}\left(x^{\prime}-\varepsilon t, y^{\prime}\right) s_{2}(x-\varepsilon t, y) d t= \\
\int_{-\infty}^{\infty} M\left(x^{\prime}-\varepsilon t, y^{\prime}\right) M(x-\varepsilon t, y) d t \\
\int_{-\infty}^{\infty} M\left(x^{\prime}-\varepsilon t, y^{\prime}\right) M(x-\varepsilon t, y) d t=\sum \delta\left(x-x^{\prime}, y-y^{\prime}\right)+k
\end{gathered}
$$

where $\mathrm{k}$ is a constant. It then follows that:

$$
\begin{gathered}
O(x, y)=\iint_{-\infty}^{\infty} g\left(x^{\prime}, y^{\prime}\right) h\left(x-x^{\prime}, y-y^{\prime}\right) \ldots \ldots \\
\ldots \ldots\left[\sum \delta\left(x-x^{\prime}, y-y^{\prime}\right)+k\right] d x^{\prime} d y^{\prime}
\end{gathered}
$$

If that the PSF is smaller than the distance between two peaks, then:

$$
O(x, y)=g(x, y) h(0,0)+k L R I
$$

where LRI stands for "low-resolution image". This means that to get a high-resolution image, the low-resolution image must be subtracted from the reconstruction.

\section{Results and discussion}

Images of unstained thin blood smears were captured using the laboratory-assembled microscope shown in Figure 1. This was done in the transmission mode at wavelengths of $405 \mathrm{~nm}$ and $638 \mathrm{~nm}$. This choice is not fortuitous because according to the work of Dabo et al., ${ }^{16}$ these wavelengths are characteristic of the malaria parasite. Ipatov and Barker masks (filters) were both used for low-resolution image acquisition. The reconstructed "Ipatov filter" and "Barker filter" images are presented and discussed next.

\section{Wavelength $\lambda=405 \mathrm{~nm}$}

Figure 3 shows images that were acquired with $405 \mathrm{~nm}$ laser illumination. The low-resolution image of Figure 3(a) was acquired without mask projection. The image shown in Figure 3(b) was reconstructed using the Barker-filteracquired images. It is sharper and has better contrast than that of Figure 3(a) but the close up reveals that it 


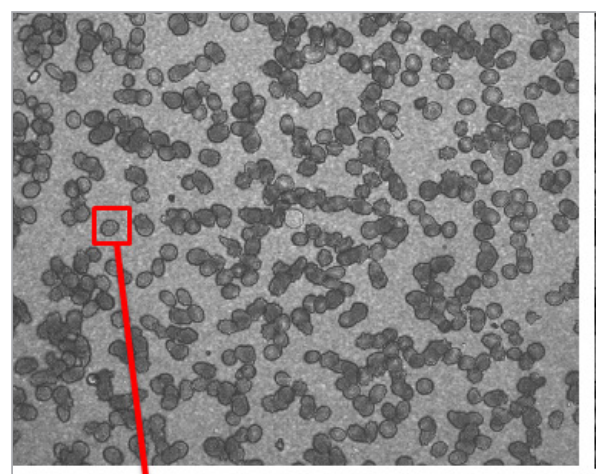

(a)

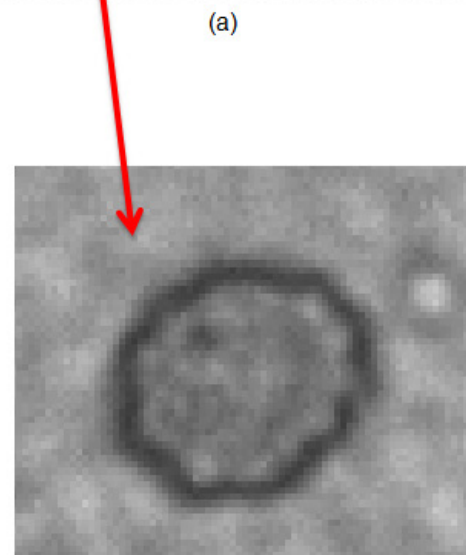

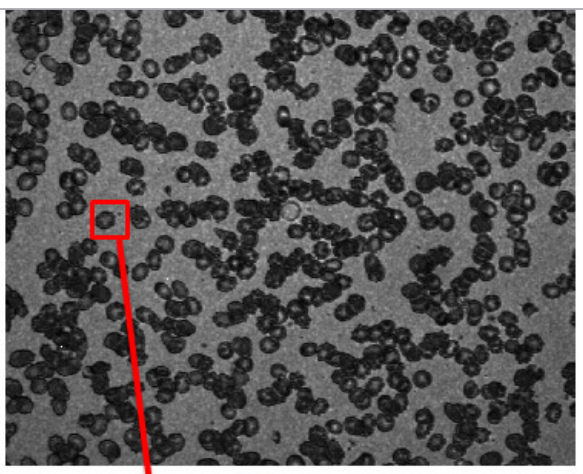

(b)

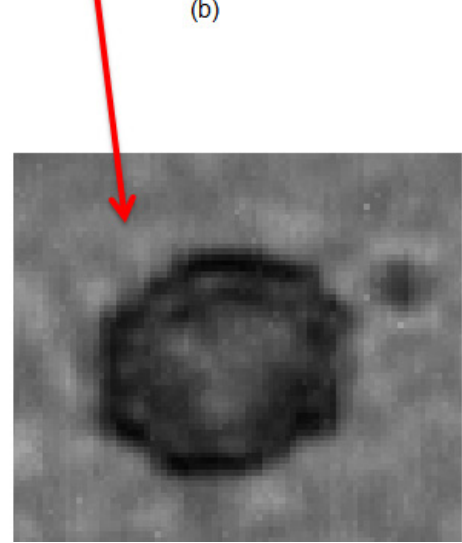

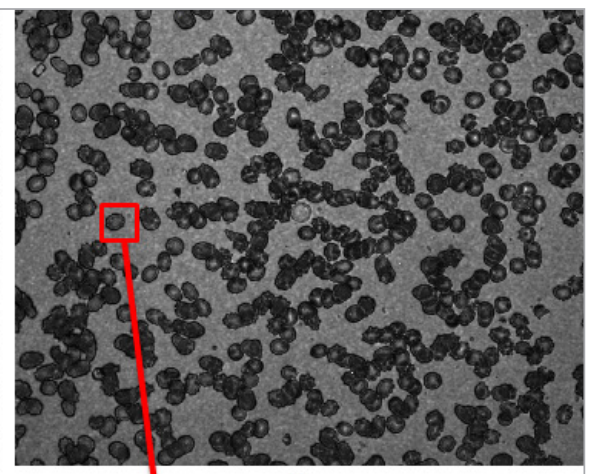

(c)

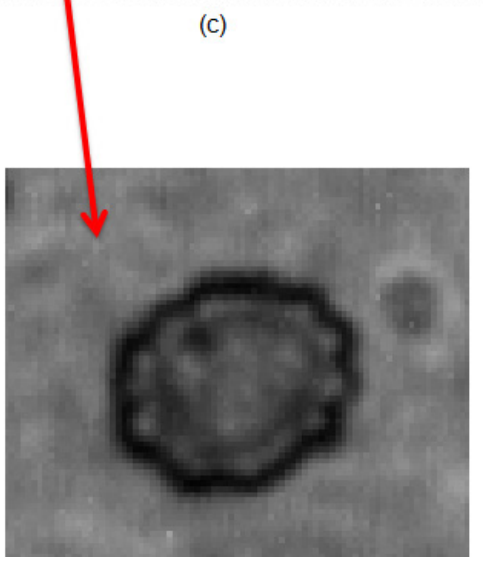

Figure 3. Transmission images using $\lambda=405 \mathrm{~nm}$. (a) Original blood smear image, (b) image of blood smear enhanced by Barker filter, (c) image of blood smear enhanced by Ipatov filter.

is blurred. Figure 3(c) shows the image reconstructed using the Ipatov-filter-acquired images. This image is clearer and the cell core is visible (see close up). It has an improved contrast, a background free of speckle and a visible cell edge.

The following metrics were computed to help with analysis of the reconstructed images:

- Mean squared error (MSE). The mean squared error between the original image and reconstructed image is expressed as:

$$
\text { MSE }=\frac{\sum_{a, b}[\mathrm{~g}(a, b)-\hat{\mathrm{g}}(a, b)]^{2}}{\sum_{a, b}[\mathrm{~g}(a, b)]^{2}}
$$

where $\mathrm{g}(a, b)$ is the original low-resolution image and $\hat{\mathrm{g}}(a, b)$ is the reconstructed super resolved image.
Peak signal-to-noise ratio (PSNR) defined as:

$$
P S N R=10 \log \left(\frac{255^{2}}{M S E}\right)
$$

- Root Mean Square Error (RMSE). RMSE $=\sqrt{M S E}$

- Standard deviation (SD)

Table 1 shows the computed parameters for reconstructed images based on Barker and Ipatov filters for $\lambda=405 \mathrm{~nm}$.

Note that PSNR for the Ipatov-filtered image is higher than that of the Barker-filtered image by a margin of $0.0331 \mathrm{~dB}$. The Ipatov-filtered image also has lower values of the errors MSE and RMSE (by $0.0812 \mathrm{~dB}$ and $0.0125 \mathrm{~dB}$, respectively). The SD of the Ipatov-filtered image is 0.0083 less than that of the Barker-filtered one. According to Singh and Goyal, ${ }^{17}$ a high value of

Table 1. Comparison of PSNR, MSE, RMSE and SD for $\lambda=405 \mathrm{~nm}$.

\begin{tabular}{|l|c|c|c|c|}
\hline Objective parameters & PSNR & MSE & RMSE & SD \\
\hline Barker filter & 37.8788 & 10.6808 & 3.2682 & 1.0950 \\
\hline Ipatov filter & 37.9119 & 10.5996 & 3.2557 & 1.1033 \\
\hline
\end{tabular}




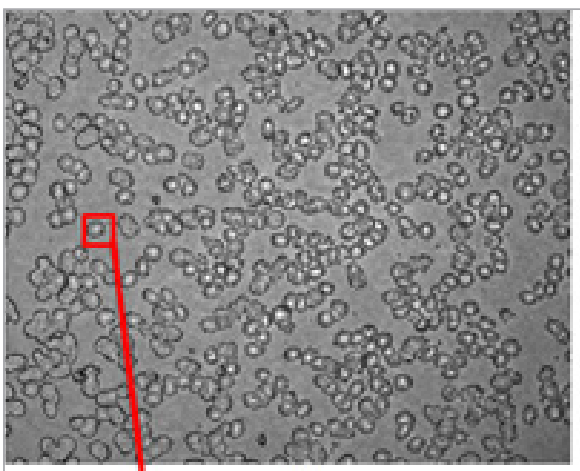

(a)

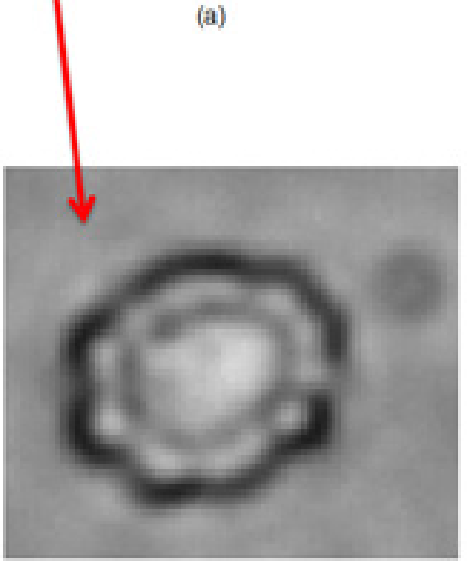

Figure 4. Transmission images using $\lambda=638 \mathrm{~nm}$. (a) Original blood smear image, (b) image of blood smear enhanced by Barker filter, (c) image of blood smear enhanced by Ipatov filter.

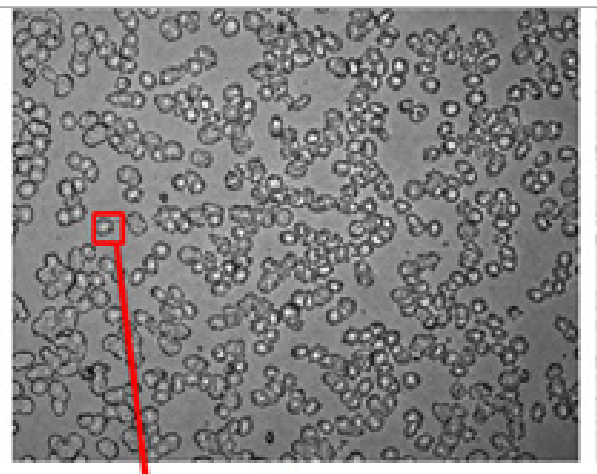

(b)

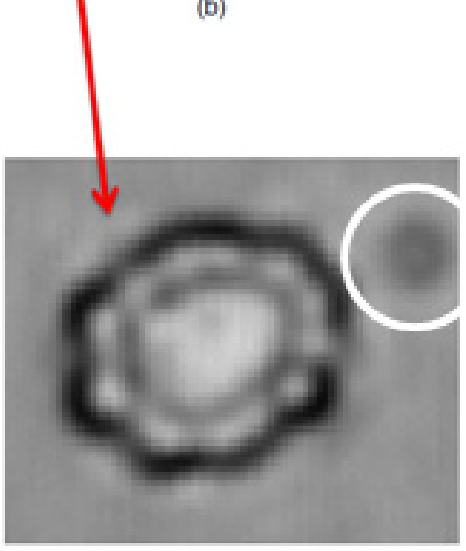

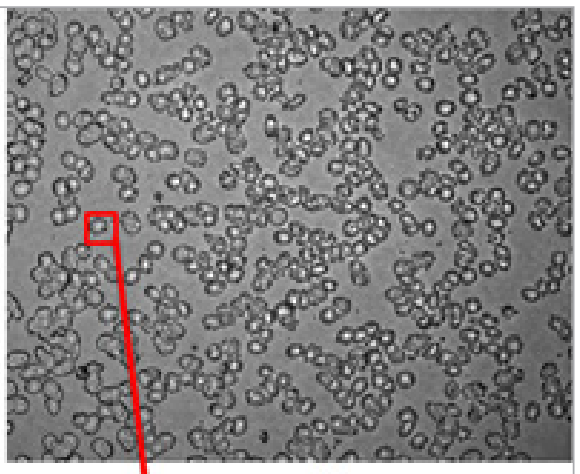

(c)

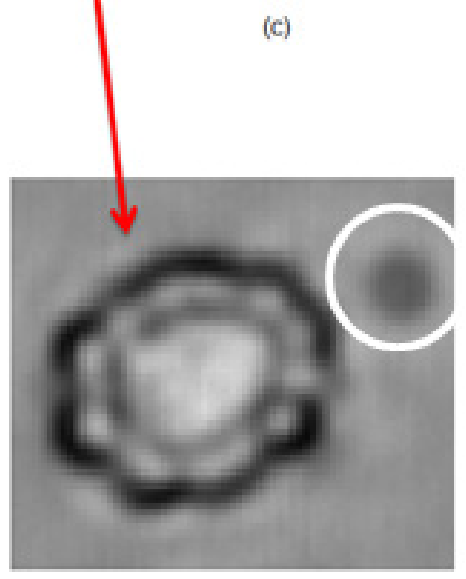

PSNR indicates that the image is more detailed. Superior quality images will have low MSE and low RMSE. A high SD suggests a high contrast in an image. The results in Table 1 are proof that the use of the Ipatov filter results in better image resolution and contrast than the Barker filter.

\section{Wavelength $\lambda=638 \mathrm{~nm}$}

Figure 4 (a) shows the low-resolution image without mask projection with illumination at $\lambda=638 \mathrm{~nm}$. The image in Figure 4(b), which was reconstructed from Barker-filter-acquired images, is sharper and has better contrast than that of Figure 4(a). Figure 4(c) shows the image reconstructed using Ipatov-filter-acquired images. This image is brighter, has visible contours and an enhanced contrast. Speckle noise has been completely eliminated.
The contrast improvement is clearly visible, not only in the white marked bars, but also in the image background. The Ipatov-filtered image has better contrast that the one that based on the Barker filter.

Table 2 shows the computed parameters for reconstructed images based on Barker and Ipatov filters for $\lambda=638 \mathrm{~nm}$.

The PSNR value of the Ipatov-filtered image is higher than that of the Barker-filtered image by a margin of $0.0292 \mathrm{~dB}$. The MSE and RMSE for the Ipatov-filtered image are also less by $0.1210 \mathrm{~dB}$ and $0.0142 \mathrm{~dB}$, respectively. The SD of the Ipatov-filtered image exceeds that of the Barker-filtered image by 0.0238 .

For both wavelengths, the reconstruction performed with an Ipatov filter gives better results than the one that uses the Barker filter because of the autocorrelation property.

Table 2. Comparison of PSNR, MSE, RMSE and SD for $\lambda=638 \mathrm{~nm}$.

\begin{tabular}{|l|c|c|c|c|}
\hline Objective parameters & PSNR & MSE & RMSE & SD \\
\hline Barker filter & 35.6150 & 17.9881 & 4.2412 & 1.2432 \\
\hline Ipatov filter & 35.6442 & 17.8671 & 4.2270 & 1.2670 \\
\hline
\end{tabular}




\section{Conclusion}

In this paper, we propose an application of TMSR to thin blood smear image enhancement. Two distinct masks, the proposed Ipatov mask and a Barker mask, were used to enhance the contrast of the reconstructed image. The masks have autocorrelation properties. The results show that both masks increase the contrast of the image, but the Ipatov filter performs better than the Barker filter. The images obtained by applying the TMSR method are without speckle noise and individual red blood cells are distinguishable. The improvement reported in this paper will result in better analyses of multispectral images. This will lead to a more accurate diagnosis of malaria, one of the leading causes of death in Africa.

\section{Acknowledgements}

We would like to thank the International Science Programme (ISP) and The World Academy of Sciences (TWAS) for financial support.

\section{References}

1. E.K. Abbe, "Beiträge zur theorie des mikroskops und der mikroskopischen wahrnehmung", Arch. Mikrosk. Anat. 9(1), 413-418 (1873). https://doi.org/10.1007/ BF02956173

2. M. Françon, "Amélioration de la reśolution d'optique", Nuovo Cimento. Suppl. 9, 283-290 (1952). https://doi.org/10.1007/BF02903399

3. W. Lukosz, "Optical systems with resolving powers exceeding the classical limit", J. Opt. Soc. Am. 56, 1463-1472 (1966). https://doi.org/10.1364/ JOSA. 56.001463

4. A. Shemer, D. Mendlovic, Z. Zalevsky, J. Garcia and P.G. Martinez, "Super resolving optical system with time multiplexing and computer decoding", Appl. Opt. 38, 7245-7251 (1999). https://doi.org/10.1364/ AO.38.007245

5. J. Garcia, Z. Zalevsky and D. Fixler, "Synthetic aperture superresolution by speckle pattern projection", Opt. Express 13, 6073-6078 (2005). https://doi. org/10.1364/OPEX.13.006073

6. D. Fixler, J. Garcia, Z. Zalevsky, A. Weiss and M. Deutsch, "Speckle random coding for 2-D super resolving fluorescent microscopic imaging", Micron
38, 121-128 (2007). https://doi.org/10.1016/j. micron.2006.07.008

7. C. Ventalon and J. Mertz, "Quasi-confocal fluorescence sectioning with dynamic speckle illumination", Opt. Lett. 30, 3350-3352 (2005). https://doi. org/10.1364/OL.30.003350

8. C. Ventalon and J. Mertz, "Dynamic speckle illumination microscopy with translated versus randomized speckle patterns", Opt. Express 14, 7198-7209 (2006). https://doi.org/10.1364/OE.14.007198

9. A. Ilovitsh, E. Preter, N. Levanon and Z. Zalevsky, "Time multiplexing super resolution using a Barkerbased array", Opt. Lett. 40(2), 163-165 (2015). https://doi.org/10.1364/OL.40.000163

10. T.J. Zoueu, G.L. Loum, C.T. Haba, B. Mikkel and H. Menan, "Optical microscope based on multispectral imaging applied to Plasmodium diagnosis", J. Appl.

Sci. 8, 2711-2717 (2008). https://doi.org/10.3923/ jas.2008.2711.2717

11. O.K. Bagui and J.T. Zoueu, "Red blood cells counting by circular Hough transform using multispectral images", J. Appl. Sci. 14(24), 3591-3594 (2014). https://doi.org/10.3923/jas.2014.3591.3594

12. M. Sangare, T.A. Agneroh, O.K. Bagui, I. Traore, A. Ba and J.T. Zoueu, "Classification of African mosaic virus infected cassava leaves by the use of multispectral imaging", Opt. Photon. J. 5, 261-272 (2015). https://doi.org/10.4236/opj.2015.58025

13. A.K. Kouakou, O.K. Bagui, T.A. Agneroh, A.P. Soro and J.T. Zoueu, "Cucumber mosaic virus detection by artificial neural network using multispectral and multimodal imagery", Optik 127(23), 11250-11257 (2016). https://doi.org/10.1016/j.ijleo.2016.09.035

14. WHO, Basic Malaria Microscopy Learner's Guide. World Health Organization, Geneva (2010).

15. N. Levanon and E. Mozeson, Radar Signals. Wiley (2004). https://doi.org/10.1002/0471663085

16. S. Dabo-Niang and J.T. Zoueu, "Combining kriging, multispectral and multimodal microscopy to resolve malaria-infected erythrocyte contents", J. Microsc. 247, 240-251 (2012). https://doi.org/10.1111/ j.1365-2818.2012.03637.x

17. G. Singh and G. Goyal, "Artificial neural networks for single-image super-resolution", Int. J. Comput. Appl. 122(16), 0975-8887 (2015). https://doi. org/10.5120/21786-5075 